\title{
Excitatory Connection from Lateral Hypothalamic Self-Stimulation Sites to Escape Sites in Medullary Reticular Formation
}

\author{
James J. Keene and Kenneth L. Casey ${ }^{1}$ \\ Department of Physiology, The Unizersity of Michigan, Ann Arbor, Michigan 48104
}

Reccived March 26, 1950

\begin{abstract}
Behavioral tests show that noncontingent stimulation of self-stimulation sites in the lateral hypothalamus increases escape and avoidance from central or peripheral stimuli. The present study investigated the possibility that this phenomenon was mediated by an excitatory connection from the lateral hypothalamus to neurons in aversive regions of the brainstem. Most of the 24 rats participated in two phases of the experiment: unit recording under anesthesia; and behavioral tests with intracranial stimulation in the waking state. Recordings of 120 units were obtained from the $n$. gigantocellularis reticularis (NGC) since stimulation of this region in the awake rat consistently elicited escape behavior. Unit responses could be divided into two catagories: $61 \%$ responded to deep pinch or fout shock (or both) but not to light brushing of the hair; and $39 \%$ responded to light brushing of the hair or were not responsive. Stimulation of behaviorally confirmed self-stimulation sites in lateral hypothalamus evoked short-latency (2 and $4 \mathrm{msec}$ ) excitation of $88 \%$ of the NGC units in the first category and only $15 \%$ in the second. Nearly all hypothalamic sites not eliciting self-stimulation also failed to excite NGC units. The excitatory hypothalamic-bulboreticular connection may serve to increase the excitability of a nociceptive system during self-stimulation.
\end{abstract}

\section{Introduction}

It is well established that many species will perform work in order to receive electrical stimulation of the lateral hypothalamus in the region of the medial forebrain bundle (MFB) (16). Brain stimulation in the awake animal has also revealed a number of sites associated with escape, avoid ance, or pain-related behavior $(5,16)$. Of the regions with possible direct hypothalamic connections, the mesencephalic and medullary reticular formation have been implicated as playing a role in aversive behavior on the basis of a number of investigations in different species. Recent neurophysiological $(1,3)$. behavioral ( 6 ; Casey, unpublished), and anatomical

${ }^{1}$ Supported by Grant NB 06588 from the National Institute of Neurological Diseases and Stroke. Dr. Keene is supported by NIH Training Grant GM 35.3. 
(9) studies have provided evidence that the medial medullary reticular formation in the region of the nucleus gigantocellularis (NGC) (17) may form part of a neural system $(1,2,11)$ subserving somatic pain.

The apparently opposing behavioral results obtained from lateral hypothalamic and reticular formation stimulation, and the anatomical connections between these areas $(8,10,13)$, raises the question of their functional relationship. At the behavioral level, investigations of approach-escape interaction have shown that aversive stimuli produce a reduction (15) or no change (4) in the rewarding properties of reinforcing brain stimuli, depending on the testing procedure. However, noncontingent stimulation of lateral hypothalamic self-stimulation sites has been shown to increase both escape from tegmental stimulation (15) and avoidance of grid shock (7). In this report, we present evidence for an excitatory connection from self-stimulation points in the lateral hypothalamus to somatically activated neurons in an aversive region of the bulboreticular formation.

\section{Methods}

Eighteen male albino rats were anesthetized with sodium pentobarbital $(40 \mathrm{mg} / \mathrm{kg})$ and placed in a stereotaxic apparatus. Figure 1 (upper) shows the experimental arrangement. Three screws were secured in the exposed skull to anchor an assembly of four to six $100-\mu$ diameter nichrome wire stimulating electrodes in the lateral hypothalamus and surrounding areas. The external surface of the plug and electrodes was completely insulated to the exposed cross section of the tips. In some cases the array was arranged in a linear sequence on one side of the hypothalamus and, in others, two twistcd pairs were dircetcd toward the MFB on cach side of the brain. The focus of the distribution of stimulating points in the lateral hypothalamus was $3.5 \mathrm{~mm}$ posterior to the bregma, $1.5 \mathrm{~mm}$ lateral to the midsaggital suture, and $8.5 \mathrm{~mm}$ from the horizontal skull surface. Constant current stimulators were used to deliver pulses to pairs of stimulating electrodes in the hypothalamus and to bipolar needle electrodes inserted subcutaneously in the pad of the contralateral hind paw.

Stainless-steel microelectrodes were used to explore the $\mathrm{N}$ GC region11-12 $\mathrm{mm}$ pasterior from bregma, $0-1 \mathrm{~mm}$ lateral from the midsaggital suture, and $8-9 \mathrm{~mm}$ from the skull surface at the point of insertion. Occasionally, two such electrodes were lowered simultaneously, each with its own channel of amplification and oscilloscope display.

During microelectrode exploration, the hind limb was stimulated at $1 \mathrm{~Hz}$ by means of the subcutaneous needle electrodes at currents just sufficient to elicit slight flexion in the lightly anesthetized preparation. When a unit was encountered, a variety of nalural sonatic stimuli (brushing hair, light 


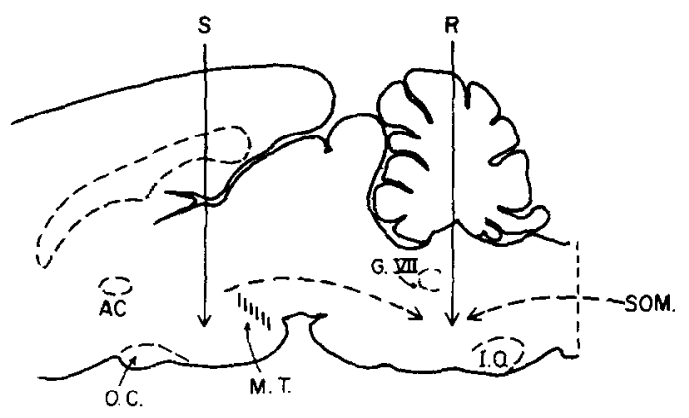

A.
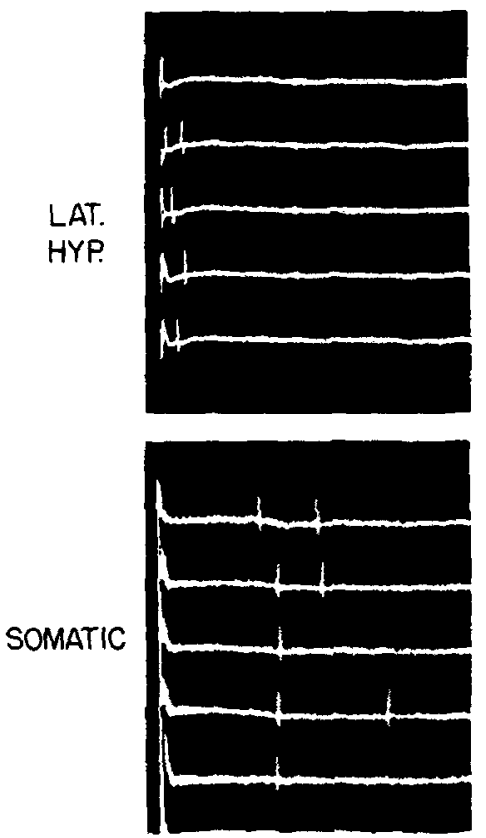

B
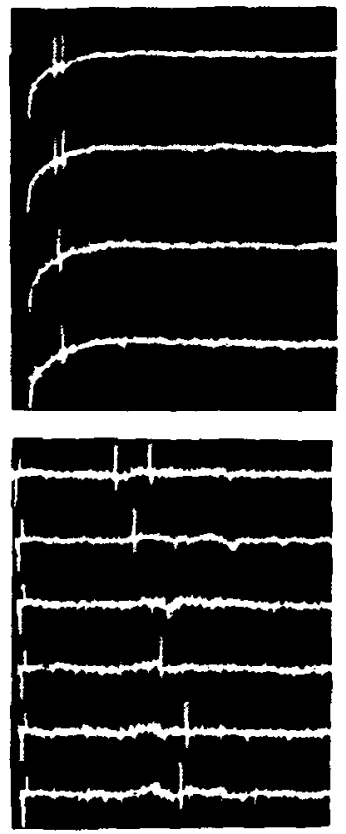

FIG. 1. Above, experimental arrangement. Units recorded ( $R$ ) in medullary reticular formation were tested for response to somatic (SOM) and hypothalamic (S) stimulation. Ac, anterior commissure: OC, optic chaism; MT, mammalothalamic tract; G VII, genu of VII nerve; IO, inferior olive. Below, examples of NGC unit responses (A and $B)$ to both lateral hypothalamic and subeutaneous foot (SOMATIC) stimulation. Unit A: lateral lypothalamic stimulus elicits 2- to 4-msec latency response; foot stimulus, 16-nisec latency response. Lnit B: lateral hypothalamic stimulus, 4-msec latency discharge: foot stimulus, response with latency variable between 14 and $25 \mathrm{msec}$.

or heavy pressure, brisk tapping, and deep pinch) were routinely employed. Clicks, handelaps, and diffuse illumination of the syes were used to test for auditory and visual imput. 
Each unit was also tested for response to each of the stimulating cathodes in the hypothalamic area. Unit responsiveness was tested at 1 and also at $60 \mathrm{~Hz}$ so that comparison could be made with subsequent behavioral data. Each bulboreticular unit included in the responsive category followed the 0.5 -msec hypothalamic pulses on at least 8 of 10 trials within a narrow latency range $(2-3 \mathrm{msec})$. The response latency and thresholdstimulating current were recorded in each case; most units were photographed for further analysis. Figure $1 \mathrm{~A}$ and $\mathrm{B}$ shows examples of unit responses to both hypothalamic and hind-limb stimulation.

When each microelectrode was estimated to have reached the ventral border of NGC, it was raised, if necessary, to the center of the region to which units responding to both somatic and hypothalamic stimuli were found, and cemented in position for chronic stimulation at the recording site.

After a week or more of recovery, behavioral tests were performed on the same animals for hypothalamic self-stimulation, escape elicited by NGC stimulation, and escape elicited by NGC stimulation with and without noncontingent hypothalamic stimulation. The rats were tested in $6 \times 12$-in. chambers provided with a bar for operant control of stimulus delivery. Stimuli consisted of $0.5-\mathrm{msec}$ square pulses at $60 \mathrm{~Hz}$ for both hypothalamic and reticular formation stimulation. Hypothalamic stimuli were delivered to each pair of electrodes in the array in separate tests; reticular formation stimulation was cathodal, one of the skull screws being anodal.

A hypothalamic cathode was classified as eliciting self-stimulation if the animal achieved at least 20 presses/min in a 5 -min test with 0.3 -sec trains of stimulation given on a continuous reinforcement schedule at current levels in the same range as those effective in driving NGC units in the acute part of the experiment. In the escape test, a lever press turned off a constant train of bulboreticular stimuli for $6 \mathrm{sec}$. An NGC cathode was considered to elicit escape behavior if the rat turned off the stimuli for more than half of a 5 -min test period. The NGC current was then decreased to a subthreshold level at which the animal made no effort to escape. A hypothalamic cathode was considered to facilitate NGC escape when the addition of noncontingent hypothalamic stimulation would then elicit escape. Eight of the rats used in the acute phase of this experiment were tested for escape responses to medial bulboreticular stimulation. Six additional rats were each prepared with two pairs of bipolar stimulating electrodes implanted in or near NGC for additional tests of escape responses.

The electrode tips were histologically located in $40-\mu$ sections of appropriately perfused and fixed brains alternately stained for cell bodies and fibers (Fig. 2). 


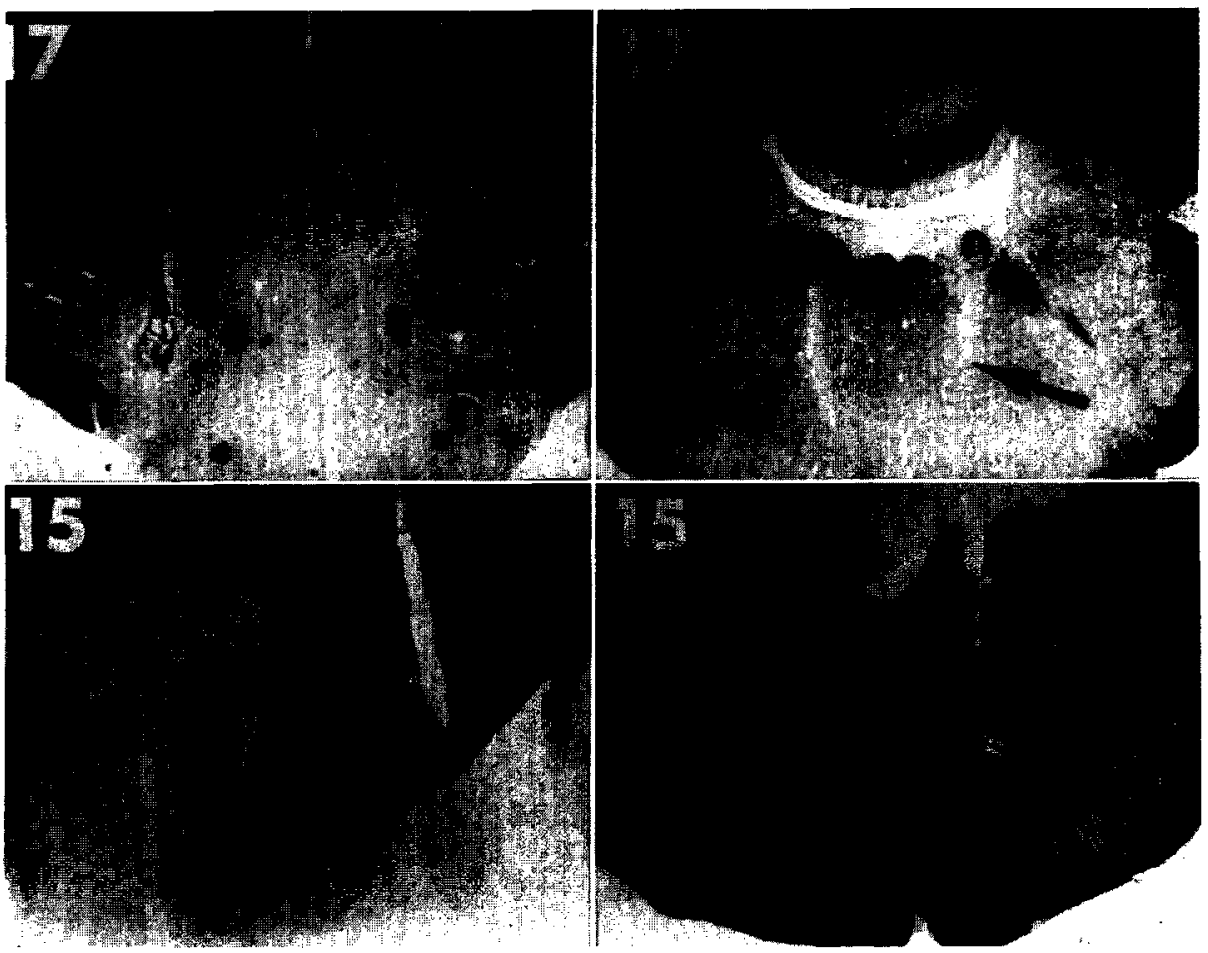

FIG. 2. Histological location of electrode positions in rats 17 and 15 . Right; NGC units recorded at the arrows were excited by deep pinch and foot shock, but not by light tactile stimulation. Stimuli delivered to electrodes in lateral hypothalamus (17, left) produced short-latency excitation of these $\mathrm{NGC}$ units (17, right) while electrodes outside lateral hypothalamus (15, left) did not excite NGC units (15, right) or elicit self-stimulation. For rat 17 , the hypothalamic electrode pair to the left elicited a high rate of self-stimulation; the pair to the right was not tested.

\section{Results}

Responses of NGC Units to Somatic Stimuli. A total of 120 units was recorded and the somatic responsiveness of 107 of these was examined. Sixty-five per cent of the 107 were regularly excited by foot shock at latencies of $10(48 \%)$ and $15(24 \%)$ msec. Fifty-five per cent responded to deep pinch but not to light brushing of the hair. Twenty-four per cent responded to light brushing of the hair and 21 per cent were not responsive to natural somatic stimulation. All responsive units had large and diffuse receptive fields such as an entire limb or the whole body surface. Table 1 shows that those units excited only by deep pinch are more likely to be excited by electrical stimulation $\left(\chi^{2}=18.64, p<10^{-4}\right)$.

Figure 3 shows the relation between response to somatic stimuli and anatonical location. Analysis of the anatomical distribution of these units 
TABLE 1

Relation Between Pinch and Foot-Shock Responsiveness

Responsive to:

\begin{tabular}{lcc} 
& Pinch, not hair & $\begin{array}{c}\text { Hair, or } \\
\text { nonresponsive }\end{array}$ \\
\hline $\begin{array}{l}\text { Driven by } \\
\text { foot stimulation }\end{array}$ & 35 & 8 \\
$\begin{array}{l}\text { Not driven by } \\
\text { foot stimulation }\end{array}$ & 7 & 16 \\
\hline
\end{tabular}

reveals that those responding only to electrical or strong cutaneous stimuli are more likely to be encountered in a region the dorsal border of which is $1 \mathrm{~mm}$ below the floor of the fourth ventricle (see arrows in Fig. 3, $\chi^{2}=24, p<10^{-\bar{s}}$ ) within the $\mathrm{n}$. gigantocellularis. Unresponsive units, or those responding to hair movement, tend to be distributed within the dorsal part of the medial medullary reticular formation.

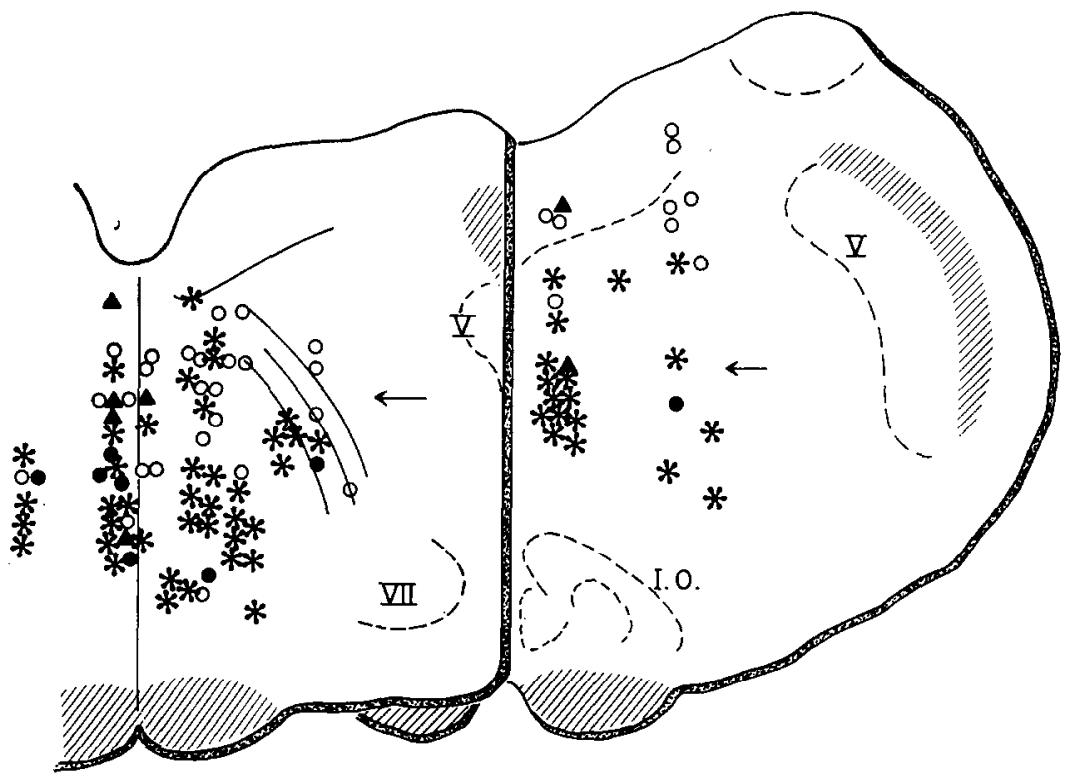

FIG. 3. Anatomical location of NGC units. Asterisks and filled triangles represent units excited by pinch or foot shock (or both), but not by light tactile stimuli. Most of these units were also excited by lateral hypothalamic stimuli (asterisks). Circles represent units which were either unresponsive or excited by hair stimulation; the majority of these (open circles) were not excited by lateral hypothalamic stimuli. 
Responses of NGC Units to Hypothalamic Stimulation. Stimulation within the lateral hypothalamus excited NGC units at latencies of 2-3 msec; threshold currents ranged from 50 to $600 \mu$ a. Single pulses or trains of pulses were never observed to decrease the frequency of ongoing NGC unit activity or to block NGC responses to somatic stimuli. The excitatory effect of lateral hypothalanic stimulation was confirmed by the oluservation that simultaneous subthreshold somatic and hypothalamic stinuli elicited unit discharge at a latency of $10 \mathrm{msec}$.

Figure 4 shows the anatomical location of the hypothalamic stimulating points. Analysis of the distribution of electrode positions reveals that the sites from which bulboreticular units were excited are more likely to be located in the lateral hypothalamus, lateral to the lateral horder of the fornix and medial to the medial edge of the cerebral peduncle $\left(x^{2}=9.8\right.$, $p<.005)$. Of the 17 stimulating points which failed to excite any reticular units, 12 were located ontside this lateral hypothalamic region.

Inspection of Fig. 3 and Table 2 reveals the relation between responsiveness to lateral hypothalamic and somatic stimulation. Thus, 58 of the 65 bulboreticular units responding only to electrical or strong cutaneous stimuli were also excited by lateral hypothalamic stimulation which was ineffective for 34 of the remaining 42 cells $\left(\chi^{2}=54, p<10^{-12}\right)$.

Units excited by hypothalamic stimulation were also excited at the highest stimulus frequency tested $(60 \mathrm{~Hz})$ at currents equal to or slightly less

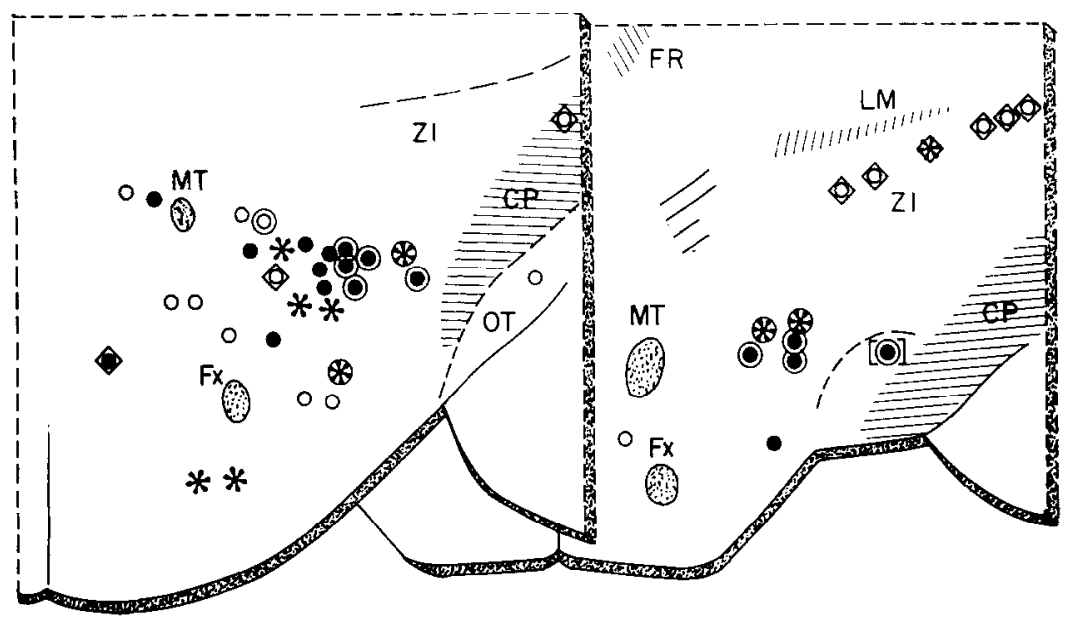

FIG. 4. Hypothalamic stimulation sites. Symbols enclosed in large circles indicate self-stimulation sites; those enclosed in diamonds show regions failing to elicit selfstimulation. Excitation of NGC units was obtained with stimulus currents below $200 \mu \mathrm{a}$ (asterisks) or between 200 and $600 \mu \mathrm{a}$ (filled circles). (Bracketed point was in substantia nigra.) Small open circles indicate failure to excite $\mathrm{NGC}$ units. 
TABLE 2

Relation Between lateral Hypothalamic and Somatic Responsiveness

\begin{tabular}{lcc}
\hline & \multicolumn{2}{c}{ Responsive to: } \\
\cline { 2 - 3 } & $\begin{array}{c}\text { Pinch and/or foot } \\
\text { shock, not hair }\end{array}$ & $\begin{array}{c}\text { Hair, or } \\
\text { nonresponsive }\end{array}$ \\
\hline $\begin{array}{l}\text { Excited by lateral } \\
\text { hypothalamic stimuli } \\
\begin{array}{l}\text { Not excited by lateral } \\
\text { hypothalamic stimuli }\end{array}\end{array}$ & 58 & 8 \\
\hline
\end{tabular}

than those required at lower frequencies. Higher stimulus frequencies were not employed since the latency, variation in latency, and response probabilities did not suggest antidromic activation from the hypothalamus.

Hypothalamic Self-Stimulation. Twenty-six hypothalamic electrode sites were tested for self-stimulation, using currents well within the range used during the acute phase of the experiment. All but one of the 14 points yielding self-stimulation were located within the lateral hypothalamus as defined above; the single exception was located in the dorsal substantia nigra (Fig. 4). Five rats had maximum response rates above $60 / \mathrm{min} ; 5$, above 40 ; and 4 , above 20 . Of the 12 electrodes which failed to yield selfstimulation, one was located within the lateral hypothalamus; the remainder were in the medial hypothalamus, cerebral peduncle, or ansa lenticularis (Fig. 4). Three electrode placements were not histologically localized. The rate of bar pressing in these 12 cases was well below $1 / \mathrm{min}$.

A significant association between self-stimulation and excitation of bulboreticular units might be expected since both effects were elicited most frequently with lateral hypothalamic electrodes. Indeed, all but one of the 14 electrode positions yielding self-stimulation excited bulboreticular units in the acute phase of the experiment. In contrast, only 2 of the 12 sites failing to elicit self-stimulation excited bulboreticular units. These data, then, establish a significant relationship $\left(x^{2}=15.8, p<10^{-4}\right)$ between hypothalamic self-stimulation sites and excitation of units in the medial medullary reticular formation.

Stimulation of Medullary Reticular Formation. Eight of the rats used in the acute phase of the experiment were also tested for response to stimulation within the NGC area. In these cases, the recording microelectrode was fixed in position for monopolar cathodal stimulation within the recording area; the skull screw used as anode failed to elicit behavioral effects when switched to cathodal polarity. As a further check on the effects of bulboreticular stimulation, six additional rats were prepared with twisted pairs of bipolar stimulating electrodes implanted bilaterally 
in the same brain-stem region. Stimulus parameters were identical to those used for hypothalamic stimulation and the currents tested ranged up to 1 ma during screening for stimulus-related behavioral effects.

Stimulation within the bulboreticular formation was usually associated with increased motor activity. Most often, this took the form of scratching and exploratory movements throughout the chamber occasionally accompanied by vocalization. In some cases, the brainstem stimulation invariably produced turning in one direction or facial movements such as flattening of vibrissae or narrowing of the palpebral fissures. However, the evoked motor activity, if any, bore no necessary relation to escape behavior since it was elicited at points failing to yield escape and was absent in several cases in which escape behavior was produced.

Figure 5 shows the location of medullary sites at which the escape criterion was reached at the current levels indicated. With the exception of one electrode pair placed near the trigeminal nucleus, the placements yielding escape at the lowest stimulating current levels were located within the NGC. Electrodes placed more dorsally, medially, or laterally were either ineffective or required currents of $200 \mu \mathrm{a}$ or more. It is quite possible

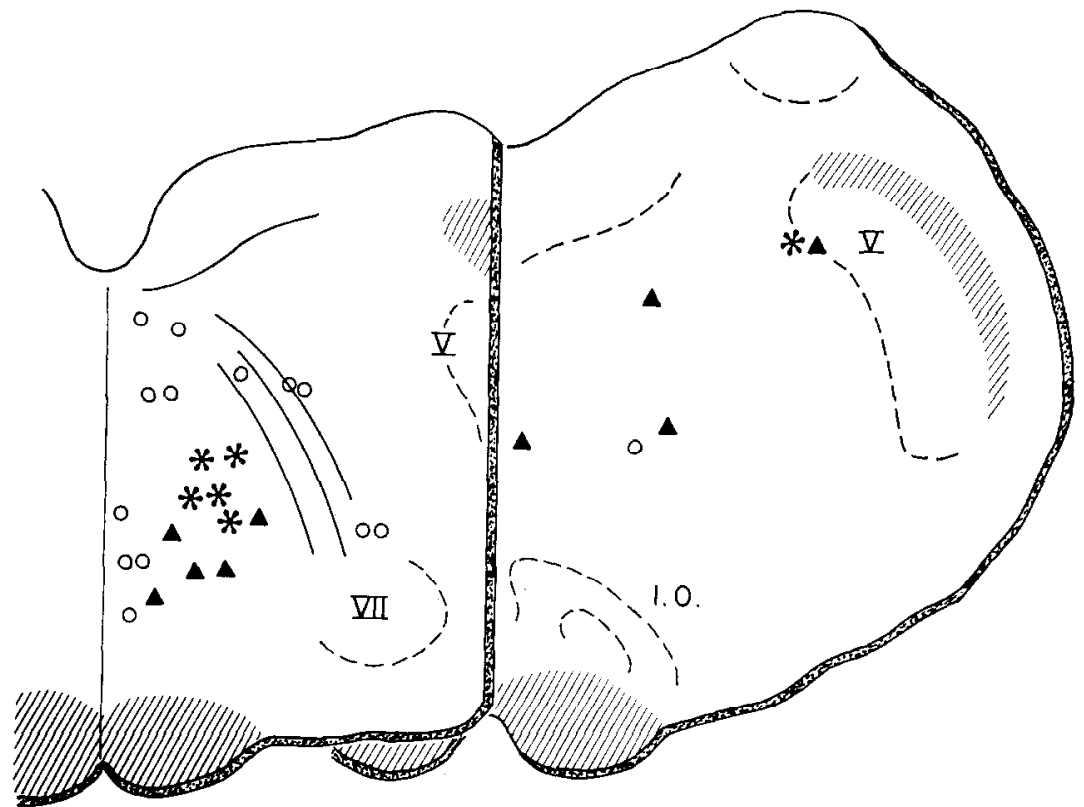

FIG. 5. Sites eliciting escape in NGC. Asterisks: attained escape criterion (see Methods) at current levels less than or equal to $100 \mu \mathrm{a}$. Filled triangles: attained escape criterion at less than or equal to $200 \mu$ a. Open circles: failed to attain escape criterion (testing currents up to $1000 \mu \mathrm{a}$ ) or reached criterion with currents greater than $200 \mu$ a. 
that these differences are due to variations among electrodes or animals and do not reflect functional differences among the various locations tested. Nonetheless, the data indicate that stimulation within the same region from which we recorded in the acute phase of this study elicits escape behavior.

Noncontingent Hypothalamic Stimulation. The electrophysiological results as well as the observations reported by others $(5,7)$ suggested that noncontingent lateral hypothalamic stimulation should facilitate the escape elicited by bulboreticular stimulation. This possibility was tested for seven hypothalamic placements which excited bulboreticular unit activity and seven which were ineffective. The NGC stimulus current was set at approximately one-half the threshold for escape behavior and the noncontingent hypothalamic stimulus parameters were identical to those used during the acute phase of the study. Stimulation of all seven of the hypothalamic sites from which bulboreticular units could be excited combined with subthreshold NGC stimulation to elicit escape. In contrast, only one of the seven placements failing to drive medullary units was effective in lowering the threshold for NGC escape.

\section{Discussion}

These experiments have demonstrated, at the level of unit analysis, an excitatory connection from hypothalamic self-stimulation loci to a somatically activated medial bulboreticular area which yields escape behavior when electrically stimulated. The results complement and extend the observations of several other investigations on the anatomical $(8,10,13)$ and functional (14) connections of hypothalamic areas considered important in motivational mechanisms. Certain features of the experiments, however, preclude a strict comparison with the results obtained by others (18). The unit sample, for example, is restricted to the medial bulboreticular area where hypothalamic stimuli evoked consistent, short-latency unit responses. In addition, an effort was made to use comparable hypothalamic stimulus parameters in both the acute and behavioral phases of the experiment, permitting a more direct comparison of the electrophysiological and behavioral data obtained in the same animal.

The neurophysiological and behavioral correlation suggested by the results depends on the identity of the neural population affected in the two phases of the study. Any correlation of this sort holds only to the extent that the bulboreticular and hypothalamic elements stimulated in the behavioral phase of the study are identical to the population studied during the acute experiments. There is no assurance that the above conditions were satisfied in this study even though similar hypothalamic stimulus parameters were used. Furthermore, there is the additional possibility that electrical stimulation of the bulboreticular formation inhibits those 
neurons receiving excitatory hypothalamic input. In view of these possible difficulties, it is, perhaps, especially surprising to find a significant correlation between some of the physiologically and behaviorally determined properties of these brain regions. The hypothalamic self-stimulation loci, for example, were not only more likely to excite bulboreticular units, but were also more likely to lower the threshold for escape responses elicited by bulboreticular stimulation. This same medullary area had the highest proportion of units responding only to deep pressure or firm pinching of the skin, a property which lends support to anatomical (9), physiological (3), and behavioral (6) evidence that this area may form part of a nociceptive system.

With the above reservations in mind, one can consider the functional significance of an excitatory influence from hypothalamic "reward" regions to a bulboreticular region of opposite behavioral valence. The inportance of this consideration is reinforced by recent studies $(7,12,15$, 21) showing that noncontingent stimulation of lateral hypothalamic selfstimulation sites facilitates escape reactions and that lesions in the farlateral hypothalamus greatly reduce hoth escape and avoidance responses to foot-shock (20). Although Cox and Valenstein (4) concluded that lateral hypothalamic stimulation attenuates the aversive properties of peripheral shock, their results seem to bear more directly on the maintenance of the self-stimulation phenomenon in the presence of aversive somatic stimuli.

Other investigators have suggested a mutually inhibitory neurophysiological relationship between areas of opposite hehavioral valence $(1+, 16$. 22); hut so far as lateral hypothalamic influences are concerned, both electrophysiological and behavioral data might also suggest excitatory connections to aversive brainstem regions $(7,12,15,19,21)$. The excitatory hypothalamic-bulboreticular connections revealed in this study may act upon reticular neurons with motor, sensory, or arousal functions related to rewarding intracranial stimuli. In view of the evidence that some bulboreticular population sulsserves aversive functions, the excitatory lateral hypothalamic influence might be regarded as part of a mechanism which increases the excitability of a nociceptive system during self-stimulation.

\section{References}

1. Bohus, B., and D. DE WIEn. 1967. Avoidance and escape behavior following medial thalamic lesions in rats. J. Comp. Physiol. Psychol. $64: 26-29$.

2. Bowsher, D., A. Mallart, D. Petit, and D. Albe-Frssard. 1968. A bulbar relay to the contre median. $I$. Neurophysinl. $31: 288-300$.

3. CAssy, K. L. 1969. Somatic stimuli, spinal pathways, and size of cutaneous fibers influencing unit activity in the medial medullary reticular formation. Exp. Nourol. 25 : $35-56$. 


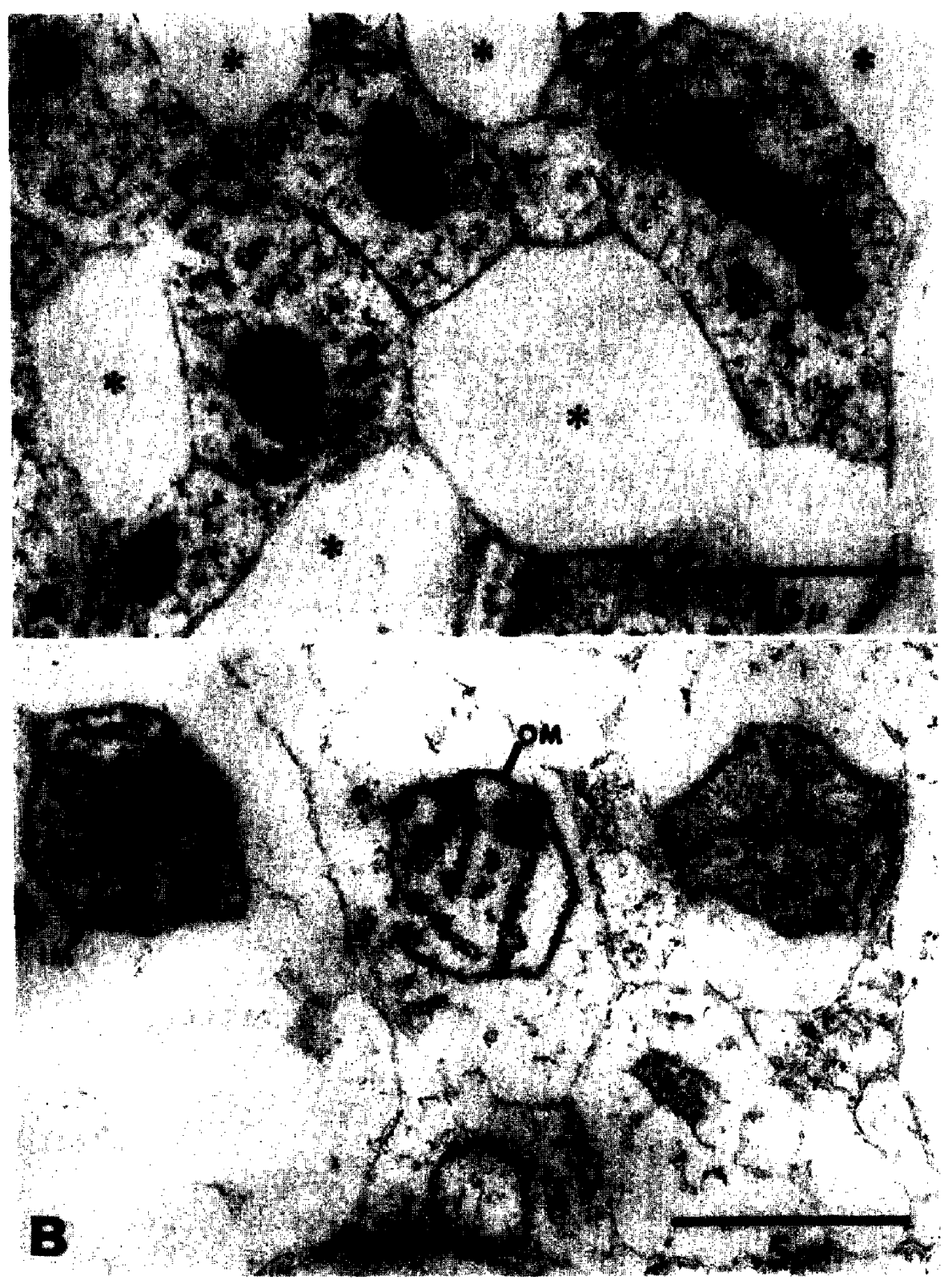

FIG. 2. Electron micrographs of near-term monkey cerebral cortex, fixed by freezesubstitution. Nitochondria are characterized by five layered inner (IM) and outer (OM) mitochondrial membranes. Extracellular spaces are identified by (*). $x$ 60,000 . A. In mitochondria of normal animals the inner-cristal matrix appears extremely dense. Cristae (IM) appear as two light lines separated by a clense line. P. In swollen mitochondria of animals subjected to prolonged partial asphyxia the inner cristal matrix is attenuated and the cristae (IMI) appear as two dense lines separated by a line of intermediate density. 